
\title{
28 Research Square \\ Linking Neuroinflammation and Extracellular Free Water in HIV Infection: A Longitudinal Study
}

Md Nasir Uddin ( $\square$ nasir_uddin@urmc.rochester.edu )

University of Rochester https://orcid.org/0000-0002-5887-1322

Abrar Faiyaz

University of Rochester

Lu Wang

University of Rochester

Yuchuan Zhuang

University of Rochester

Kyle D Murray

University of Rochester

Maxime Descoteaux

Universite de Sherbrooke

Madalina E. Tivarus

University of Rochester

Miriam T. Weber

University of Rochester

Jianhui Zhong

University of Rochester

Xing Qiu

University of Rochester

Giovanni Schifitto

University of Rochester

\section{Research}

Keywords: Free water, neuroinflammation, HIV, cART, brain, grey matter, white matter, cognition, blood markers

Posted Date: December 28th, 2020

DOI: https://doi.org/10.21203/rs.3.rs-121836/v2

License: (9) This work is licensed under a Creative Commons Attribution 4.0 International License. Read Full License 
Page $2 / 19$ 


\section{Abstract}

Initiation of combination antiretroviral therapy (cART) reduces inflammation in HIV-infected (HIV+) individuals. Recent studies demonstrated that diffusion MRI based extracellular free water (FW) modeling can be sensitive to neuroinflammation. Here, we investigate the FW in HIV-infection, its temporal evolution, and its association with blood markers, and cognitive scores. Using 96 age-matched participants, we found that FW was significantly elevated in grey and white matter in cART-naïve HIV+ compared to healthy controls (HIV-) at baseline. Similarly, at baseline, HIV+ participants had increased neurofilament light chain ( $\mathrm{NfL}$ ) values that correlated with FW and CD4 counts. FW in grey and white matter, as well as NfL decreased in the HIV+ after 12 weeks of CART treatment. No significant FW differences were noted between the HIV+ and HIV- cohorts at 1 and 2-year follow-up. Results suggest that FW elevation in CART-naïve HIV+ participants is likely due to neuroinflammation. The correlation between FW and NfL and the improvement in both FW and NfL after 12 weeks of CART treatment further reinforces this conclusion. The longer follow-up at 1 and 2 years suggests that cART helped control neuroinflammation as inferred by FW. Therefore, FW could be used as a biomarker to monitor HIVassociated neuroinflammation.

\section{Introduction}

Despite the successful suppression of viral replication and improved immune function with combination antiretroviral therapy (cART), approximately $30-50 \%$ of HIV-infected individuals still develop HIVassociated neurological disorders, including cognitive impairment ${ }^{1}$. There is evidence that inflammation persists despite undetectable viral loads, and it is thought to be the primary mechanism behind HIV disease progression and chronicity, and comorbidities such as HIV-associated cognitive impairment ${ }^{1,2}$. Microglia and perivascular macrophages are the primary actors of neuroinflammation ${ }^{3}$. These cell types are also the primary source of productive HIV infection ${ }^{4,5}$. Quantifying the presence of HIV brain infection, neuroinflammation and neuronal injury associated with the infection, are key factors in evaluating the HIV reservoir, the burden of neuroinflammation and consequences of such neuroinflammation. Evaluation of brain HIV infection in HIV-infected individuals is currently limited to indirect measurements such as those performed using cerebrospinal fluid (CSF). However, CSF viral load is undetectable in $>90 \%$ of well-treated individuals with undetectable plasma viral load, thus, CSF viral load may not adequately represent the extent of intra-parenchymal infection ${ }^{6}$.

To date, neuroinflammation due to HIV infection has been assessed using several neuroimaging biomarkers, including elevated glial markers via magnetic resonance spectroscopy (MRS) ${ }^{7,8}$, increased glucose metabolism via Positron Emission Tomography (PET) and increased microglial activation via translocator protein (TSPO) PET tracers ${ }^{9-11}$. While PET ligands offer a more direct quantification of microglia activation ${ }^{12}$, there are several drawbacks. PET imaging is expensive, it exposes individuals to significant ionizing radiation that could limit frequent scanning, ligands are not always widely available, and it is non-informative on how the signal captured is reflective of tissue damage. Specifically, in cART 
treated individuals, the expected level of activated microglia is small and the signal captured does not provide information on the effect on neurons ${ }^{13}$. In contrast, the FW index is an emerging diffusion MRI metric that indirectly and noninvasively measures the leakage of extracellular fluid into brain regions. FW index has been found to be associated with neuroinflammatory responses, atrophy, vascular risk factors and cognitive declines ${ }^{7,13-15}$. While the exact interpretation of the FW index is still unclear, one of the hypotheses is that excessive extracellular FW stems from the leakage of the blood brain barrier (BBB) with leaking serum proteins that cause axonal damage and demyelination. It is well established that the BBB plays a crucial role in the pathogenesis of the HIV-infection ${ }^{16}$.

Free water (FW) imaging, a diffusion MRI post-processing method, has been used to differentiate the extracellular non-flowing free water from the contribution of water that diffuses in proximity to the axons 17. The FW index, a measure of the relative fraction of freely diffusing water in the extracellular space (ECS), can reveal neuroaxonal damage that affects the tissue diffusion characteristics. FW has been found to be correlated with neuroinflammation and neurodegeneration in several neurological disorders 18-22. The relevance of FW as a marker of inflammation has been further evaluated by correlating FW with levels of microglia activation measured by TSPO via a PET study ${ }^{23}$. Furthermore, previous animal studies ${ }^{24-26}$ have shown that neuroinflammation alters brain ECS.

The main goals of this work were to investigate whether increased FW index would be observed in cART naïve HIV-infected individuals and if it improves with cART treatment, thus providing a marker of responsiveness to treatment. We also posited that if FW index were indicative of inflammation, it would be correlated with other makers of brain injury such as neurofilament light chain (NfL) and markers of immune function such as CD4 count.

\section{Methods And Materials}

\section{Participants}

Forty-four treatment-naïve HIV+ participants (4 females and 40 males; mean age \pm standard error, SE $=34.48 \pm 1.95$ years, range, 20 - 63 years) and 52 age-matched HIV-healthy controls ( 26 females and 26 males; mean age $\pm \mathrm{SE}=37.02 \pm 1.66$ years, range, 18-63 years) were enrolled and followed between 2013 to 2019 prior to and then following CART treatment. The study was reviewed and approved by the Institutional Research Subjects Review Board (RSRB) at the University of Rochester Medical Center. All participants provided written informed consent before enrollment, and then underwent clinical, laboratory, neurocognitive and brain MRI exams. All experiments were carried out in accordance with relevant guidelines and regulations. HIV+ participants were evaluated at baseline prior to initiation of cART and at three follow-up timepoints [12 weeks $(n=38), 1$ year $(n=29)$, and 2 years $(n=19)$ ] after cART treatment had been started, while the HIV- participants were evaluated at the baseline, 1-year $(n=46)$, and 2-year ( $n$ = 19) time points. The 12-week evaluation of the HIV-participants were not included in the study design as significant changes in MRI and clinical measures in healthy controls are not expected within such a 
short period. Detailed baseline demographics (including age, sex, clinical characteristics) are presented in Table 1.

\section{Data Acquisition}

\section{Blood sample}

Plasma levels of Neurofilament light chain (NfL) were measured by Simoa assay via a commercial lab (QuanterixTM, Lexington, MA, United States). Other clinical HIV parameters such as CD4 counts and viral loads (VL) were collected from the patient reports or standard laboratory testing that was part of this study.

\section{Neuropsychological assessments}

Trained staff supervised by a clinical neuropsychologist administered the following cognitive battery: tests of executive function (Trailmaking Test Parts A \& B, Stroop Interference task), speed of information processing (Symbol Digit Modalities Test and Stroop Color Naming), attention and working memory (CalCAP(CRT4), learning [Rey Auditory Verbal Learning Test (Trials 1-5), Rey Complex Figure Test Immediate Recall], memory (Rey Auditory Verbal Learning Test Delayed Recall, Rey Complex Figure Test Delayed Recall), verbal fluency (Controlled Oral Word Association Test), and motor function (Grooved Pegboard, left and right hand) were administrated at each visit for all participants. Premorbid intellectual functioning ability was estimated via WRAT-4 Reading at the baseline visit only. Each of the tests within a cognitive domain were normalized and averaged to obtain a domain specific z-score. These were then summed to generate a total cognitive score. HIV-associated neurocognitive disorders (HAND) diagnoses were determined for each participant based on the Frascati criteria ${ }^{27}$.

\section{Magnetic Resonance Imaging.}

MRI was performed on a 3T scanner (MAGNETOM Trio and Prisma Fit, Siemens, Erlangen, Germany). The T1-weighted (T1w) images were acquired using a 3D magnetization prepared rapid acquisition gradientecho (MPRAGE) sequence with inversion time $=1,100 \mathrm{~ms}$, repetition time $(T R)=2,530 \mathrm{~ms}$; echo time (TE) $=3.44 \mathrm{~ms}$; flip angle $=7^{\circ}$; Field of View $($ FOV $)=256 \times 256 ;$ GRAPPA $=2$; number of slices $=192$; voxel size $=1.0 \times 1.0 \times 1.0 \mathrm{~mm}^{3}$. Diffusion weighted images (DWI) were acquired using a single shot spin echo echoplanar imaging (SE-EPI) sequence with the following scan parameters: 60 diffusion-encoded images $(b=$ $\left.1000 \mathrm{~s} / \mathrm{mm}^{2}\right), 10$ non-diffusion weighted reference images $\left(b=0 \mathrm{~s} / \mathrm{mm}^{2}\right)$; TR = 8,900 ms; TE = $86 \mathrm{~ms}$; FOV $=256 \times 256 ;$ GRAPPA $=2$; number of slices $=70 ;$ number of volumes $=61 ;$ voxel size $=2.0 \times 2.0 \times 2.0$ $\mathrm{mm}^{3}$. In order to correct for EPI distortions, a double-echo gradient echo field map sequence was also acquired $\left(\mathrm{TR}=400 \mathrm{~ms} ; \mathrm{TE}=5.19 \mathrm{~ms} ; \mathrm{FOV}=256 \times 256\right.$; flip angle $=60^{\circ} ;$ number of slices $=70$; voxel size $=$ $\left.2.0 \times 2.0 \times 2.0 \mathrm{~mm}^{3}\right)$. 


\section{Data Analysis}

Image analyses were performed using a combination of image processing tools and scripting languages, including: FSL (https://fsl.fmrib.ox.ac.uk/fsl/fs/wiki/28, ANTs (http://stnava.github.io/ANTs/) ${ }^{29}$, MATLAB (version 2018b) and Python (version 3.7.4).

All MRI images were checked for any severe artifacts such as gross geometric distortion, bulk motion, and signal dropout. DWI images were corrected for eddy current-induced distortion, inter-volume participant motion, and susceptibility-induced distortion using the "topup" and "eddy" tools in FSL ${ }^{30,31}$. A bi-tensor model was used to create FW maps on a voxel-by-voxel basis from the DWI data using a previously described algorithm ${ }^{21}$ and the processing was carried out using Nextflow ${ }^{32}$ pipeline with all software dependencies bundled in a Singularity Container ${ }^{33}$. The FW values are in the range of 0 to 1 . Values approaching 0 correspond to negligible FW diffusion in the ECS while 1 corresponds to maximum FW diffusion (i.e., water in a voxel that diffuses completely freely). All the T1w images were co-registered to the MNI space $(1 \mathrm{~mm})$ using ANTs ${ }^{29}$. The same transformations were applied to the FW maps. Since the data was collected either with Siemens TIM Trio or Siemens Prisma due to scanner upgrade, we used ComBat algorithm to harmonize data across scanner for the DWI derived FW index for each $\mathrm{ROI}^{34}$. An empirical Bayes framework is used in the ComBat algorithm to estimate additive and multiplicative scanner effects.

The FW values were calculated from whole Grey Matter (GM) and White Matter (WM) using corresponding masks. In addition, the Harvard-Oxford (subcortical) and JHU-ICBM (WM and tracts) atlases were used to calculate region averages in standard space $(1 \mathrm{~mm})$ in 25 pre-defined regions-ofinterest (ROI) relevant to HIV infection ${ }^{35,36}$ (shown in supplementary tables). FW values were averaged over bilateral ROIs (except some WM tracts) for each participant.

Statistical analyses were performed in R 3.6.2 (R Foundation for Statistical Computing, Vienna, Austria). Marginal comparisons between two independent groups were conducted by either two-group Welch's unequal variances t-test (for continuous variables) or Fisher exact test (for categorical variables). Paired $t-$ tests were used to compare the levels of continuous variables in the HIV+ and HIV-participants between baseline and follow-up visits. Pearson correlation tests were used to test the marginal associations between two continuous variables. A p-value of $<0.05$ was considered statistically significant for a single hypothesis testing problem. For inferential problems that involved multiple hypotheses, the BenjaminiHochberg multiple testing procedure was used to control the false discovery rate (FDR) at the $<0.05$ level 37.

Linear mixed effects regression modeling: Due to the longitudinal nature of the data, most multivariate analyses performed in this study were based on linear mixed effects regressions (LMER), with perparticipant random intercepts to account for serial correlations between multiple time points. Empirical evidence shows that the CART treatment effect was most prominent in the first 12 weeks; its long-term effect was subtler and, in some cases, different from its short-term effect. Therefore, we performed two 
separate LMER analyses to study the longitudinal associations between covariates (such as HIV status and cART treatment) and the response variables (such as FW index and blood markers).

The Short-Term Model (STM) was applied to data collected at baseline (both HIV+ and HIV- participants) and Week-12 (HIV+ participants only). Covariates included were HIV status (cohort), short-term cART treatment (visit), and age.

The Long-Term Model (LTM) was applied to data collected from the HIV-participants at baseline and the HIV+ participants at week-12 (used as the new baseline time point in LTM), year-1 and year-2 data collected from both cohorts. Covariates included were HIV status, visit, age, and the interaction between HIV status and visit to account for possibly different temporal patterns of a response variable between two cohorts.

For both models, the parameters were estimated by the restricted maximum likelihood (REML) criterion, and the statistical significance was assessed by the adjusted ANOVA F-test and regression t-test provided by $\mathrm{R}$ package ImerTest ${ }^{38}$.

\section{Results}

\section{Participant Characteristics}

Demographic and clinical data of the participants are presented in Table 1. Welch's Two Sample t-test indicates that there is no statistically significant age difference between the HIV-and HIV+ participants ( $p$ $=0.324)$. However, we included age as a covariate in all multivariate regression analyses to control for their remaining confounding effects. Compared to HIV+, HIV- participants had a higher number of males than females $(p<0.001)$ and higher education levels $(p=0.039)$ at baseline.

\section{Baseline FW comparison for HIV+ vs. HIV-cohorts}

Figure 1A-B represents the comparisons of FW between the HIV+ and HIV- at baseline for GM and WM. Mean voxel-by-voxel FW map from HIV+ cohort is shown in Figure 1C. Marginal comparisons based on Welch's two-sample t-test showed that FW index was higher in GM ( $t=4.74$, adjusted $p$-value $\left.p_{a d j}<0.001\right)$ and in WM ( $\left.t=2.11, p_{a d j}=0.038\right)$ in the HIV+ cohort than in HIV- cohort (Figure 1, Table 1).

We also compared the FW in both cohorts categorized by age such as young adults (age $£ 30,22 \mathrm{HIV}+, 18$ HIV- participants) and older adults (age $>30,15 \mathrm{HIV}+, 32 \mathrm{HIV}$ - participants). FW was significantly higher in GM ( $\left.\mathrm{t}=10.51, \mathrm{p}_{\mathrm{adj}}<0.001\right)$ and WM $\left(\mathrm{t}=4.072, \mathrm{p}_{\mathrm{adj}}<0.001\right)$ in the HIV+ young adult than HIV-young adult. In contrast, FW was significantly higher only in $\mathrm{GM}\left(\mathrm{t}=2.589, \mathrm{p}_{\mathrm{adj}}=0.007\right)$ in the HIV+older adult compared to HIV- older adults.

Among 25 ROIs (See Supplementary Table S1) that included subcortical GM structures and WM tracts, 3 ROIs (Thalamus, Amygdala and Hippocampus) had significantly higher FW in the HIV+ cohort than in 
HIV- cohort $(p<0.05)$.

\section{Baseline comparisons of blood marker for HIV+ vs. HIV-cohorts}

Welch's two group t-test showed that average $\mathrm{NfL}$ concentration was marginally higher in HIV+ compared to the HIV- cohort ( $t=2.10, p=0.042)$ (Table 1 ).

\section{Short Term effects of cART on FW}

In paired comparisons between baseline and week-12 for HIV+ participants $(n=31$ with measures at both baseline and week-12), we found that the FW index decreased significantly in GM ( $\left.t=4.57, p_{a d j}<0.001\right)$ and $W M\left(t=2.60, p_{a d j}=0.014\right)$ (Figure 2A-B). This was also reflected in ROI based analyses (Supplementary Table S2).

Based on the STM that modeled the cohort and treatment effect simultaneously and adjusted for the confounding effects of age, we found that GM and WM are associated with significant higher FW in the $H I V+$ cohort $\left(b_{\text {cohort } G M}=0.044, p_{a d j, G M}<0.001 ;\left(b_{\text {cohort }, W M}=0.005, p_{a d j, W M}=0.004\right)\right.$. Even after accounting for the effects of HIV and treatment, age was significantly associated with the increase of FW for most of the ROls. More information on individual ROIs can be found in SupplementaryTable S3.

\section{Short Term effects of cART on blood markers}

Paired t-test showed that the average NfL concentration decreased $(t=1.61, p=0.115)$ after 12 weeks of CART treatment (Figure 2C).

\section{Long Term effects of CART on FW}

Paired t-tests revealed that FW increased in WM and GM at year-1 compared to week-12 and baseline for the HIV+ and HIV- cohorts respectively $(p<0.05)$. However, at year-1 and year-2 visits there were no significant differences in GM and WM between the HIV+ and HIV- (Figure 3A-B).

The LTM showed there were no significant cohort effect and the interaction of cohort and visit in GM and WM suggesting that levels of FW in the HIV+ cohort were stabilized after 12 weeks of cART treatment (Figure 3A-B). The LTM on ROls also revealed similar results as presented in Supplementary Table S4. Age is invariably associated with significant increase of FW.

\section{Long Term effects of CART HIV+ on NfL}

The average NfL concentrations were found to become stable after 12 weeks (new baseline) of cART treatment and follow-up visits in the HIV+ cohort. Similar to FW, after 12 weeks of cART treatment, no significant differences in NfL were found between the HIV+ and HIV-cohorts during the follow-up visits (Figure 3C). 
Pearson correlation analysis for HIV+ at baseline showed that average $\mathrm{NfL}$ concentration was correlated with $\mathrm{FW}$ in $\mathrm{GM}$ and WM ( $\left.\mathrm{b} \gg 0.6, \mathrm{p}_{\mathrm{adj}}<0.001\right)$. In addition, we found significant positive correlations for $\mathrm{FW}$ vs. NfL in 19 of 25 ROls. The CD4 cell counts were negatively associated with FW in GM and WM (b»-0.4, $\mathrm{p}_{\mathrm{adj}}=0.041$ ), as well as in 14 out of 25 ROls. However, no significant correlations were found for $\mathrm{FW} \mathrm{vs.} \mathrm{VL}$ for any ROIs. Details are provided in Supplementary Table S5.

The total cognitive score was also lower in the HIV+ cohort compared to the HIV-cohort at baseline $(p=0.028)$. Paired t-test showed that total cognitive score increased after 12 weeks of cART treatment in $\mathrm{HIV}+$ cohort $(\mathrm{p}<0.001)$. After 12 weeks of cART treatment and follow-up visits in HIV+ cohort, total cognitive scores became stable. The total cognitive score was not significantly correlated with FW for WM and GM in both cohorts at baseline; however, there was a trend toward a negative correlation in the GM $(r=-0.26, p=0.11)$. In the STM and LTM, FW in WM and GM were not significantly associated with the total cognitive scores. Visit and interaction between cohort and visit were found significant for FW in WM and GM in the LTM while visit was significant in the STM $\left(\mathrm{p}_{\mathrm{adj}}<0.05\right)$.

\section{Discussion}

In this study, we used the FW index to indirectly assess neuroinflammation associated with HIV infection. The rationale to use FW as a putative marker of inflammation is based on previous studies in other brain diseases ${ }^{18-21,23,26}$. We reasoned that HIV infected cART naïve participants would be at higher risk of inflammation (higher FW) and that treatment would reduce inflammation (lower FW). We also hypothesized that active neuroinflammation would be associated with higher plasma levels of $\mathrm{NfL}$ and lower cognitive performance. Four main findings emerged from this work: 1) FW index and NfL were higher in cART naïve HIV+ compared to the HIV- participants at baseline; 2) FW index and NfL decreased dramatically in GM and WM after 12 weeks of cART treatment in the HIV+;3) FW levels were comparable between the HIV+ and HIV- at 1 year and 2 years of follow-up and similar trends were observed in NfL; and 4) the baseline measures of FW index in GM and WM in the HIV+ were strongly associated with NfL concentration.

To the best of our knowledge, this is the first study to investigate FW as a possible biomarker for neuroinflammation in the HIV+ cohort. Prior work demonstrates the involvement of subcortical GM and WM structures in neuronal damage in the HIV+ participants $7,35,36,39,40$. We found significantly higher FW index in GM and WM in cART-naïve HIV+ compared to the HIV- participants at baseline, consistent with previous work in other neurological disorders ${ }^{15,18-21,41}$. Increased FW in GM and WM in the HIV+ cohort might be related to abnormal neuroimmune response 42,43 .

The plasma level of the Neurofilament light chain (NfL) is a promising blood marker for neuroaxonal degradation ${ }^{44}$. NfL is considered a direct measure of neuronal damage since it is released into the brain's ECS following axonal injury and consequently into the CSF and blood ${ }^{44,45}$. Elevated NfL levels are observed in several neurological and neurodegenerative disorders including HIV+ cohort ${ }^{41,45-47}$. In this 
work, the average NfL concentration was significantly higher, by 39\%, in the HIV+ compared to HIVparticipants. The FW index was found to be positively correlated with $\mathrm{NfL}$ and negatively correlated with CD4 counts. Several previous studies suggested that low CD4 counts might be linked to brain atrophy including cortical thinning, volume reduction in GM and WM as well as ventricular enlargement ${ }^{48-50}$. As such, low CD4 is associated with elevated extracellular FW in brain tissue. These results provide additional evidence that FW may be related to neuroinflammatory processes. However, in the present study we did not find an association between FW and VL.

A significant finding was that FW decreased drastically in whole GM and WM and several ROIs in the brain after 12 weeks of cART treatment. That is, FW was decreased significantly by $21 \%$ and $8 \%$ in the GM and WM respectively due to the cART treatment. After 12 weeks of CART treatment, FW values were close to those of the HIV- participants implying that short term CART treatment normalized FW in the HIV+ cohort. In contrast, the average NfL concentration was reduced by $~ 17 \%$ after 12 weeks of cART treatment in the HIV+ cohort. A previous study also reported that $\mathrm{NfL}$ levels decreased to normal level after 6 months of CART initiation in acute HIV-infection ${ }^{51}$. These findings indicate that the change in neuroinflammation due to the short-term treatment effect can be estimated using FW index.

After the initial changes due to 12-weeks of cART exposure, no significant differences in FW were observed between the HIV + and HIV-participants during the two years of follow-up. However, we found that FW increased significantly at year-1 compared to that of baseline in the HIV-participants and compared to 12 weeks of cART treatment in the HIV+ participants. Comparison of young adults and older adults revealed that these differences in FW were driven more by the young adults (age $<30$ years) in both cohorts. It is not clear whether a yearly change in FW water should be expected as both of our cohorts showed no further changes at year-2 of follow-up although it is worth to notice that the sample size was much reduced at year-2 follow-up compared to year-1. Further investigation with large sample is required to confirm these findings. Overall, FW and NfL exhibited similar temporal trends over 2 years follow-up visits, suggesting that after CART initiation there is likely minimal level of neuroinflammation.

Of notice, there was no significant correlation between cognitive performance and FW. It is possible that changes in cognitive performance require some neuronal and glia structural damage while FW dynamics can happen at any stage including those where cellular integrity is minimally altered. The trend observed in negative correlation between cognitive performance and FW may also suggest that the relatively small sample size may have contributed to the limited association.

This study has a few limitations worth considering. First, the proportion of male and female participants was not equal, however the FW index in GM and WM was not significantly different in males vs. females in our HIV-participants. Second, the number of participants was lower for follow-up visits, especially at year-2 and blood markers were not collected for all participants.

\section{Conclusions}


In summary, our findings suggest that extracellular FW is elevated in the brains of cART naïve HIV+ participants at a time when neuroinflammation is expected to be high. Higher levels of $\mathrm{NfL}$ and its correlation with FW tend to support this possibility. Most importantly, short term cART treatment effectively reduces the levels of FW and stabilizes it over the 2 years of follow-up. Although the biological underpinnings of the elevated FW index are still unclear, FW is a potential inflammatory marker that could be used to monitor disease course and response to interventions in HIV-infected individuals.

\section{Abbreviations}

$\mathrm{FW}=$ free water; $\mathrm{FWI}=$ free water imaging; $\mathrm{NfL}=$ neurofilament light chain; $\mathrm{cART}=$ combination antiretroviral therapy; $\mathrm{DTI}=$ diffusion tensor imaging; $\mathrm{GM}=$ grey matter; $\mathrm{WM}=$ white matter; $\mathrm{ROI}=$ region of interest; TSPO=translocator protein; PET = Positron emission tomography; ECS = extracellular space; VL = viral load; SE = standard error; HAND = HIV-associated neurocognitive disorders; MPRAGE = magnetization prepared rapid acquisition gradient-echo; DWI = diffusion weighted imaging; RSRB = Research Subjects Review Board; TR = repetition time; TE = echo time; FOV = field of view; FDR = false discovery rate; LMER = linear mixed effects regressions; STM = short-term model; LTM = long-term model; $\mathrm{REML}$ = restricted maximum likelihood; $\mathrm{p}_{\mathrm{adj}}=$ adjusted $\mathrm{p}$-value; $\mathrm{BBB}=$ blood brain barrier; $\mathrm{MRS}=$ magnetic resonance spectroscopy.

\section{Declarations}

\section{Acknowledgments}

This study was supported by grant from the NIH (R01-MH099921, R01 AG054328, and R01-MH118020). We are greatly indebted to the patients and healthy volunteers for participating in this study.

\section{Author contributions}

MNU: Conceptualization, image processing, data analysis and interpretation, and manuscript writing; AF: Image processing and manuscript review for intellectual content; LW: Statistical analysis; YZ: Data collection and quality check, and manuscript review for intellectual content; KDM: Data collection and manuscript review for intellectual content; MD: Software, and manuscript review for intellectual content; MET: Manuscript review for intellectual content; MTW: Cognitive data collection and manuscript review for intellectual content; JZ: Manuscript review for intellectual content; XQ: Statistical analysis and interpretation, and manuscript review for intellectual content; GS: Project administration, funding acquisition, study concept and interpretation, manuscript review for intellectual content. All authors have read and approved the manuscript.

\section{Additional information}

None of the authors have any competing financial or non-financial interests in relation to the work described in this manuscript. 


\section{Data availability}

Anonymized data will be made available on reasonable request, pending appropriate institutional review board approvals.

\section{Approval for human experiments}

All protocols were reviewed and approved by the Research Subjects Review Board (RSRB) at the University of Rochester Medical Center. All participants signed a written consent form.

\section{References}

1 Heaton, R. et al. HIV-associated neurocognitive disorders persist in the era of potent antiretroviral therapy: CHARTER Study. Neurology 75, 2087-2096 (2010).

2 Lederman, M. M., Funderburg, N. T., Sekaly, R. P., Klatt, N. R. \& Hunt, P. W. in Advances in immunology Vol. 119 51-83 (Elsevier, 2013).

3 Maartens, G., Celum, C. \& Lewin, S. R. HIV infection: epidemiology, pathogenesis, treatment, and prevention. The Lancet 384, 258-271 (2014).

$4 \quad$ Wallet, C. et al. Microglial Cells: The Main HIV-1 Reservoir in the Brain. Frontiers in cellular and infection microbiology 9, 362, doi:10.3389/fcimb.2019.00362 (2019).

5 Abreu, C. et al. Brain macrophages harbor latent, infectious simian immunodeficiency virus. AIDS (London, England) 33 Suppl 2, S181-s188, doi:10.1097/qad.0000000000002269 (2019).

6 Livelli, A. et al. Correlates of HIV RNA concentrations in cerebrospinal fluid during antiretroviral therapy: a longitudinal cohort study. The lancet. HIV 6, e456-e462, doi:10.1016/s2352-3018(19)30143-2 (2019).

$7 \quad$ Harezlak, J. et al. Persistence of HIV- associated cognitive impairment, inflammation and neuronal injury in era of highly active antiretroviral treatment. AIDS (London, England) 25, 625 (2011).

8 Chang, L. et al. Effects of APOE $\varepsilon 4$, age, and HIV on glial metabolites and cognitive deficits. Neurology 82, 2213-2222 (2014).

9 Hammoud, D. A. et al. Imaging glial cell activation with [11C]-R-PK11195 in patients with AIDS. Journal of neurovirology 11, 346-355 (2005).

10 Boerwinkle, A. \& Ances, B. M. Molecular imaging of neuroinflammation in HIV. Journal of Neuroimmune Pharmacology 14, 9-15 (2019).

11 Crawshaw, A. A. \& Robertson, N. P. The role of TSPO PET in assessing neuroinflammation. Journal of neurology 264, 1825-1827 (2017). 
12 Alam, M. M., Lee, J. \& Lee, S.-Y. Recent progress in the development of TSPO PET ligands for neuroinflammation imaging in neurological diseases. Nuclear medicine and molecular imaging 51, 283296 (2017).

13 Peluso, M. J. et al. Immediate initiation of CART is associated with lower levels of cerebrospinal fluid YKL-40, a marker of microglial activation, in HIV-1 infection. AIDS (London, England) 31, 247 (2017).

14 Maillard, P. et al. Cerebral white matter free water: A sensitive biomarker of cognition and function. Neurology 92, e2221-e2231 (2019).

15 Guo, J. et al. Brain free water alterations in first-episode psychosis: a longitudinal analysis of diagnosis, course of illness, and medication effects. Psychological Medicine, 1-10 (2020).

16 Avison, M. J. et al. Neuroimaging correlates of HIV-associated BBB compromise. Journal of neuroimmunology 157, 140-146 (2004).

17 Pasternak, O., Sochen, N., Gur, Y., Intrator, N. \& Assaf, Y. Free water elimination and mapping from diffusion MRI. Magnetic Resonance in Medicine: An Official Journal of the International Society for Magnetic Resonance in Medicine 62, 717-730 (2009).

18 Andica, C. et al. Free-Water Imaging in White and Gray Matter in Parkinson's Disease. Cells 8, 839 (2019).

19 Pasternak, O., Westin, C.-F., Dahlben, B., Bouix, S. \& Kubicki, M. The extent of diffusion MRI markers of neuroinflammation and white matter deterioration in chronic schizophrenia. Schizophrenia research $161,113-118$ (2015).

20 Hoy, A. R. et al. Microstructural white matter alterations in preclinical Alzheimer's disease detected using free water elimination diffusion tensor imaging. PloS one 12, e0173982 (2017).

21 Dumont, M. et al. Free water in white matter differentiates $\mathrm{MCl}$ and $\mathrm{AD}$ from control subjects. Frontiers in aging neuroscience 11, 270 (2019).

22 Wang, Q., Liu, Y. \& Zhou, J. Neuroinflammation in Parkinson's disease and its potential as therapeutic target. Translational neurodegeneration 4, 19 (2015).

23 Reid B.E., D. B. M. A., Pomper M.G., Shenton M.E., Du Y., Coughlin J.M., Pasternak O. in Proceedings of the ISMRM 27th Annual Meeting and Exhibition.

24 Lo, W. D., Wolny, A. C., Timan, C., Shin, D. \& Hinkle, G. H. Blood-brain barrier permeability and the brain extracellular space in acute cerebral inflammation. Journal of the neurological sciences 118,188 193 (1993). 
25 Febo, M. et al. Diffusion magnetic resonance imaging-derived free water detects neurodegenerative pattern induced by interferon-y. Brain Structure and Function 225, 427-439 (2020).

26 Di Biase, M. A. et al. Increased extracellular free-water in adult male rats following in utero exposure to maternal immune activation. Brain, Behavior, and Immunity 83, 283-287 (2020).

27 Antinori, A. et al. Updated research nosology for HIV-associated neurocognitive disorders. Neurology 69, 1789-1799 (2007).

28 Jenkinson, M., Beckmann, C. F., Behrens, T. E., Woolrich, M. W. \& Smith, S. M. Fsl. Neuroimage 62, 782-790 (2012).

29 Avants, B. B., Tustison, N. \& Song, G. Advanced normalization tools (ANTS). Insight j 2, 1-35 (2009).

30 Andersson, J. L., Skare, S. \& Ashburner, J. How to correct susceptibility distortions in spin-echo echo-planar images: application to diffusion tensor imaging. Neuroimage 20, 870-888 (2003).

31 Smith, S. M. et al. Advances in functional and structural MR image analysis and implementation as FSL. Neuroimage 23, S208-S219 (2004).

32 Di Tommaso, P. et al. Nextflow enables reproducible computational workflows. Nature biotechnology 35, 316 (2017).

33 Kurtzer, G. M., Sochat, V. \& Bauer, M. W. Singularity: Scientific containers for mobility of compute. PloS one 12, e0177459 (2017).

34 Fortin, J.-P. et al. Harmonization of multi-site diffusion tensor imaging data. Neuroimage 161, 149170 (2017).

35 Kamat, R. et al. Apathy is associated with white matter abnormalities in anterior, medial brain regions in persons with HIV infection. Journal of clinical and experimental neuropsychology 36, 854-866 (2014).

36 Zhu, T. et al. Patterns of white matter injury in HIV infection after partial immune reconstitution: a DTI tract-based spatial statistics study. Journal of neurovirology 19, 10-23 (2013).

37 Benjamini, Y. \& Hochberg, Y. Controlling the false discovery rate: a practical and powerful approach to multiple testing. Journal of the Royal statistical society: series B (Methodological) 57, 289300 (1995).

38 Kuznetsova, A., Brockhoff, P. B. \& Christensen, R. H. B. ImerTest Package: Tests in Linear Mixed Effects Models. J Stat Softw 82, 1-26, doi:DOI 10.18637/jss.v082.i13 (2017). 
39 Ances, B. M., Ortega, M., Vaida, F., Heaps, J. \& Paul, R. Independent effects of HIV, aging, and HAART on brain volumetric measures. Journal of acquired immune deficiency syndromes (1999) 59, 469 (2012).

40 Chang, K. et al. Plasma inflammatory biomarkers link to diffusion tensor imaging metrics in virally suppressed HIV-infected individuals. AIDS (2019).

41 Pasternak, O. et al. Excessive extracellular volume reveals a neurodegenerative pattern in schizophrenia onset. Journal of Neuroscience 32, 17365-17372 (2012).

42 Pasternak, O., Kubicki, M. \& Shenton, M. E. In vivo imaging of neuroinflammation in schizophrenia. Schizophrenia research 173, 200-212 (2016).

43 Lesh, T. A. et al. Extracellular free water and glutathione in first-episode psychosis-a multimodal investigation of an inflammatory model for psychosis. Molecular psychiatry, 1-11 (2019).

44 Teunissen, C. E. \& Khalil, M. Neurofilaments as biomarkers in multiple sclerosis. Multiple Sclerosis Journal 18, 552-556 (2012).

45 Gisslén, M. et al. Plasma concentration of the neurofilament light protein (NFL) is a biomarker of CNS injury in HIV infection: a cross-sectional study. EBioMedicine 3, 135-140 (2016).

46 Gattringer, T. et al. Serum neurofilament light is sensitive to active cerebral small vessel disease. Neurology 89, 2108-2114 (2017).

47 Zhou, W. et al. Plasma neurofilament light chain levels in Alzheimer's disease. Neuroscience letters 650, 60-64 (2017).

48 Hassanzadeh-Behbahani, S. et al. Low CD4 nadir linked to widespread cortical thinning in adults living with HIV. Neurolmage: Clinical 25, 102155 (2020).

49 Sanford, R. et al. Regionally specific brain volumetric and cortical thickness changes in HIVinfected patients in the HAART era. Journal of acquired immune deficiency syndromes (1999) 74, 563 (2017).

$50 \mathrm{Nir}, \mathrm{T} . \mathrm{M}$. et al. Progressive brain atrophy in chronically infected and treated HIV+ individuals. Journal of neurovirology 25, 342-353 (2019).

51 Peluso, M. J. et al. Absence of cerebrospinal fluid signs of neuronal injury before and after immediate antiretroviral therapy in acute HIV infection. The Journal of infectious diseases 212, 17591767 (2015).

\section{Table}


Table 1: Baseline demographic, clinical and free water (FW) data.

\begin{tabular}{|c|c|c|c|c|}
\hline Characteristics & & $\mathrm{HIV}+(\mathrm{n}=44)$ & HIV- $(n=52)$ & p-value \\
\hline Age, mean (SE) & & $34.48(1.95)$ & $37.02(1.66)$ & 0.3242 \\
\hline \multirow[t]{3}{*}{ Sex, n (\%) } & & & & $<0.001$ \\
\hline & Female & $4(9.1 \%)$ & $26(50 \%)$ & \\
\hline & Male & $40(90.9 \%)$ & $26(50 \%)$ & \\
\hline \multirow[t]{3}{*}{ Ethnicity, n (\%) } & & & & 0.408 \\
\hline & Hispanic or Latino & $4(9.1 \%)$ & $2(3.8 \%)$ & \\
\hline & Not Hispanic or Latino & 40 (90.9\%) & $50(96.2 \%)$ & \\
\hline \multirow[t]{5}{*}{ Race, n (\%) } & & & & $<0.001$ \\
\hline & Caucasian & $20(45.5 \%)$ & $42(80.8 \%)$ & \\
\hline & Black AA & $22(50 \%)$ & $5(9.6 \%)$ & \\
\hline & Other & $1(2.3 \%)$ & $4(7.7 \%)$ & \\
\hline & Missing & $1(2.3 \%)$ & $1(1.9 \%)$ & \\
\hline \multirow[t]{3}{*}{ Education, n (\%) } & & & & 0.039 \\
\hline & $\leq 12$ Years & $13(29.5 \%)$ & $6(11.5 \%)$ & \\
\hline & $>12$ Years & $31(70.5 \%)$ & $46(88.5 \%)$ & \\
\hline \multicolumn{5}{|l|}{ Blood markers } \\
\hline & $\mathrm{CD} 4{ }^{+} \mathrm{T}$ cell count $/ \mathrm{mm}^{3}$ & $509.66 \pm 41.24$ & - & - \\
\hline & Viral load (VL) copies/mL & $78236.76 \pm 20585.89$ & - & - \\
\hline & Neurofilament (NfL) pg/mL & $12.02 \pm 2.17$ & $7.32 \pm 0.56^{*}$ & 0.042 \\
\hline \multicolumn{5}{|c|}{ HAND classification, $\mathrm{n}(\%)$} \\
\hline & Normal & $21(47.7 \%)$ & $26(50.0 \%)$ & 0.3132 \\
\hline & ANI & $22(50.0 \%)$ & $21(40.4 \%)$ & \\
\hline & MND & $1(2.3 \%)$ & $5(9.6 \%)$ & \\
\hline Total Cognitive S & core & $-1.62 \pm 0.56$ & $0.21 \pm 0.59$ & 0.028 \\
\hline \multicolumn{5}{|l|}{ FW index (\%) } \\
\hline & Grey matter (GM) & $17.42 \pm 0.44$ & $13.76 \pm 0.64$ & $<0.001$ \\
\hline & White matter (WM) & $4.82 \pm 0.12$ & $4.46 \pm 0.12$ & 0.041 \\
\hline
\end{tabular}

Note: Continuous variables are summarized as Mean \pm Standard Error, categorical variables are summarized as frequency (percentages); HAND: HIV-associated neurocognitive disorders; ANI: Asymptomatic neurocognitive impairment; MND: Mild neurocognitive disorder. Significant p-values are shown as bold.

*number of HIV- participants were 15.

\section{Figures}



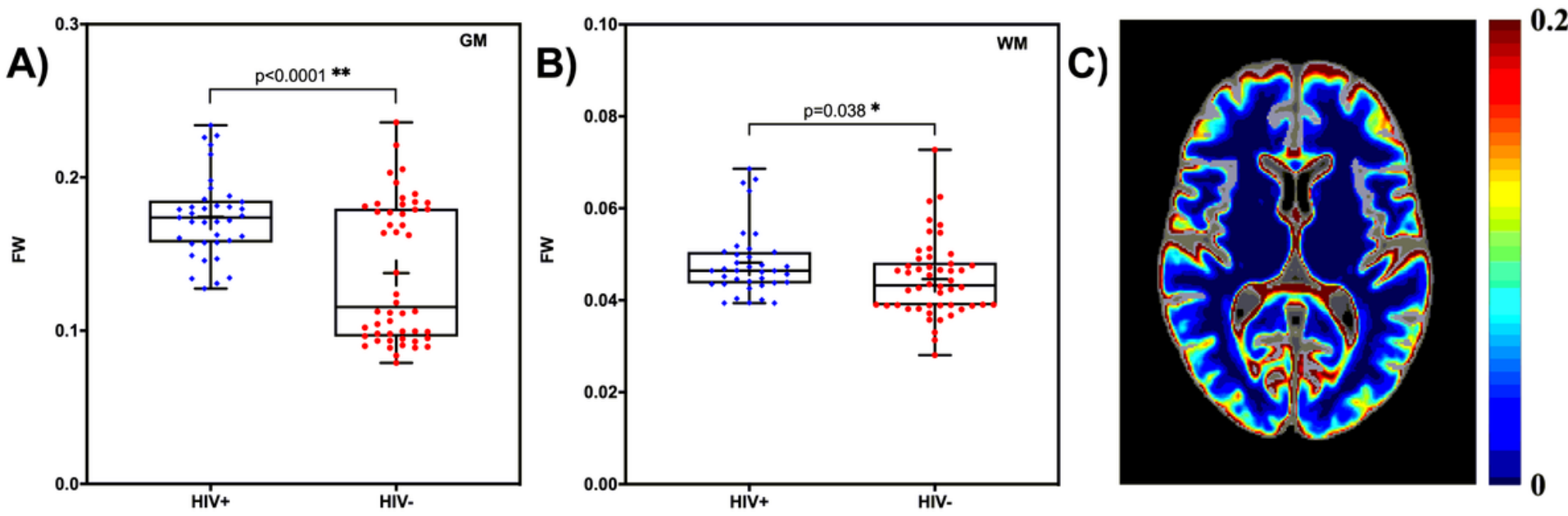

Figure 1

Baseline free water comparison for HIV+ and HIV- cohorts The box-and-whisker plots illustrate the comparison of the free water (FW) index between the HIV+ and HIV- at baseline for A) grey matter (GM) and $B$ ) white matter (WM); Note: significant differences are shown; + sign in the box plots indicates mean FW of each cohort. C) Mean FW map (axial view) from 37 HIV+ participants at baseline with intensity scale.
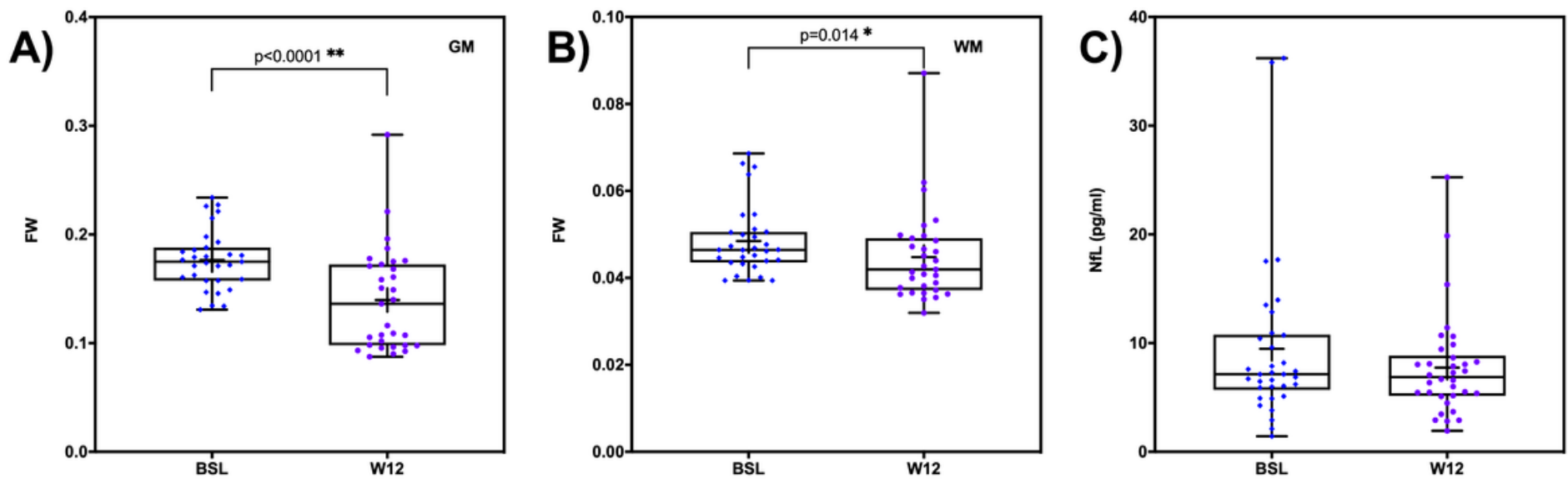

Figure 2

Short term effect of cART treatment on free water and neurofilament light chain The box-and-whisker plots illustrate the comparison of free water (FW) index in A) gray matter (GM) and B) white matter (WM); C) average neurofilament light chain (NfL) in the HIV+ participants. Note: significant differences are shown; + sign indicates mean of each measure at each time point. BSL, baseline; W12, week-12. 

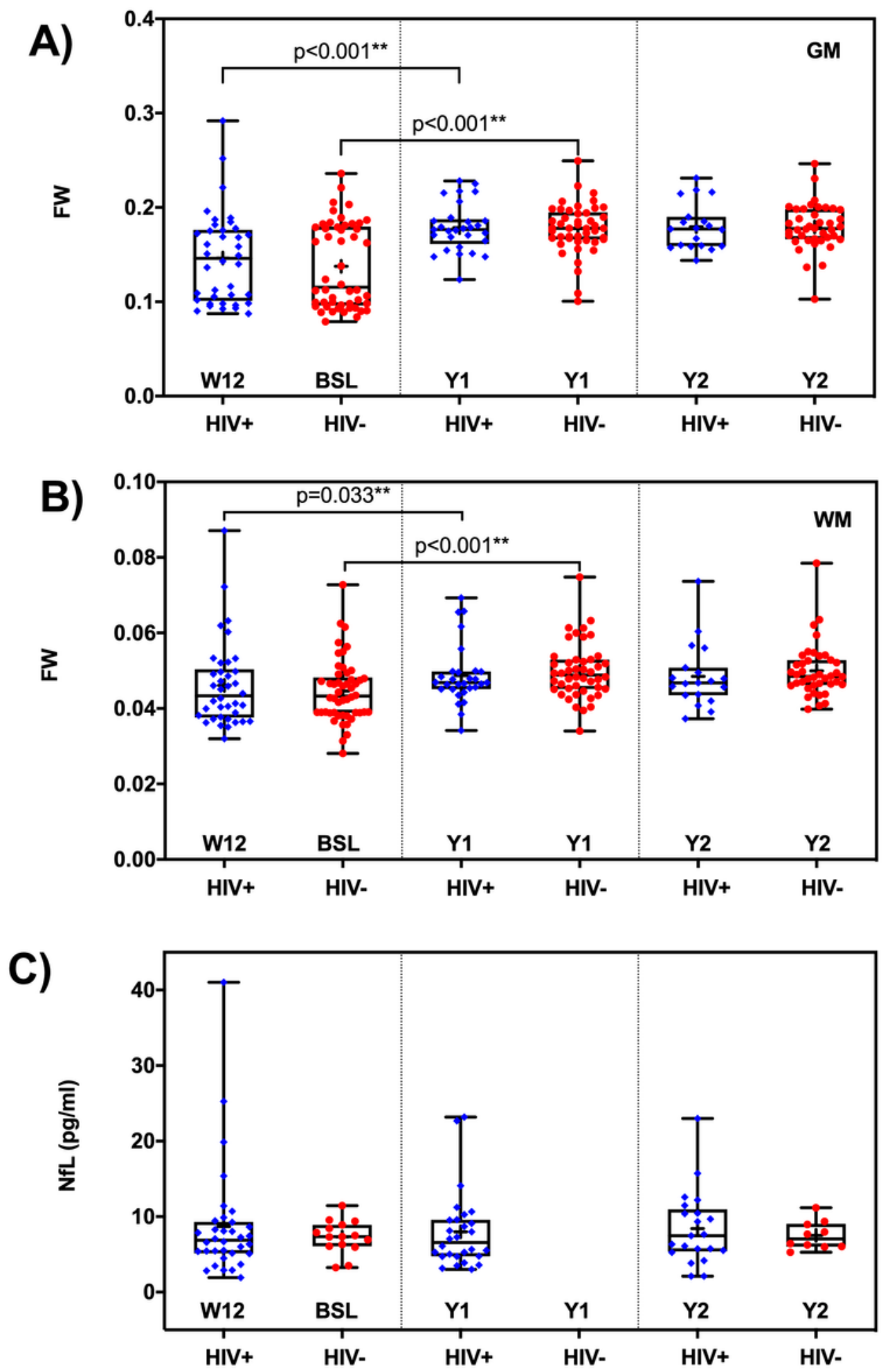

Figure 3

Long term effect of CART treatment on free water and neurofilament light chain The box-and-whisker plots illustrate the comparison of free water (FW) index between the HIV+ and HIV- participants, and the temporal trends of FW from baseline and follow-up visits in A) gray matter (GM), B) white matter (WM); C) average $\mathrm{NfL}$ concentration. There is no significant between-cohort difference of long-term temporal progression in FW (in GM and WM), and NfL. Note, BSL, baseline; W12, week-12 (new BSL for HIV+); Y1, 
year-1; Y2, year-2; NfL data were not collected at Y1 for HIV- participants. + sign indicates the mean FW at each time point and cohort. Significant differences are also shown.

\section{Supplementary Files}

This is a list of supplementary files associated with this preprint. Click to download.

- SupplementaryInformation.docx 